\title{
Comparative Analysis of Image Deblurring Techniques
}

\author{
Taresh Singh \\ Department of IT, COER Roorkee \\ Roorkee, India
}

\author{
B. M. Singh, $\mathrm{PhD}$ \\ Department of IT, COER Roorkee \\ Roorkee, India
}

\begin{abstract}
Image processing is a process to digitize the data of an image and a variety of mathematical operations are applied to enhance the image which is more applicable or pleasing to a human observer or to accomplish some of the analysis and identification tasks by computer.

Due to environmental disarray or improper camera setting blur may arise in an image. Noise can also degrade the quality of a captured image in conjunction with blur. Restoration is a process to remove the blur from the image and restore the original image.

There are a variety of techniques and methods have been suggested to restore a despoiled image. For a particular blur, there is a particular technique to remove it. In this paper, we discussed various image restoration methods and their study of efficiency.

Image restoration has a variety of applications in various fields like video surveillance, crowed movement analysis etc..

\section{Keywords}

Deconvolution, Degradation model, Point spread function (PSF), Peak signal to noise ratio (PSNR).

\section{INTRODUCTION}

Researchers focus on image deblurring is since a long time ago. A massive part of image processing area is devoted to image deblurring. Restoration method of images centred on restoration of original images from the blurred one. As noise also corrupts the image so we need to act upon image denoising. Image denoising is also a component of deblurring method. There are three main kind of blur in digital image:
\end{abstract}

\subsection{Average blur}

Average blur spreads in both directions, Horizontal and Vertical. Average filter may restore the image from this type of blur. Average filter works well when influence of noise in the entire image.

\subsection{Gaussian blur}

This blur is generated through Gaussian function. Outcome of Gaussian blur generated by a filter which is a bell-shaped curvature by unifying a definite number of pixels one by one.

\subsection{Motion blur}

If there is relative movement between the camera and the scene, motion blur may be introduced. This type of blur may be generated in an image through motion filter in a definite direction and the resulted image seems to be in motion.

\section{RELATED WORKS}

J. Biemond et al. [1] proposed recurring restoration methods for the elimination of linear blurs from images that are corrupted by point wise nonlinearities such as film saturation and additive noise.
Jubien et al. [2] proposed two different algorithms that can be combined with the neural network for blind image renovation.

J. G. Nagy et al. [3] implemented iterative Matlab tools for efficient matrix vector multiplication, and for solving the linear system for pre-conditioners, efficiently for image restoration.

P. C. Hansen et al. [4] considered a blurring model that is more robust than the previous one. The blurring is generated on the rows and columns of the image concurrently.

Z. Hongying et al. [5] analyzed some techniques that working with neural network, to converge the recorded blurred image to the sharp image.

J.Jiaya et al. [6] proposed that blurring model can be described as a blend of two operations; the first operation is convolving the original image with unknown kernel; the second operation is the addition of some noise to the consequential image.

A. Beck et al. [7] analysed a bunch of Iterative ShrinkageThresholding Algorithms (ISTA) to solve linear inverse issues for image analysis. They investigated that these methods are extremely easy but converge extremely slowly.

Cai et al.[8] presented adaptive light weight selection, which is an input in a progression of nominal sub-lexicons. It characterized each neighbourhood to repair a sub-word indication as the inadequate neighbourhood.

Et al. [9] proposed a method that is helpful for straight estimating spatially changing point multiply method. This function can't work for multiple images.

D. S. Rao et al. [10] described that Optical Transfer Function is a Fourier transfer of the point Spread Function (PSF) and the PSF is a converse Fourier transform of OTF. Inside the frequency reign, the OTF considers the reaction of a linear, position-invariant method to an inclination.

Subashini et al. [11] used highly nonlinear back propagation neuron for image renovation to get a better quality restored image and achieve fast neural computation.

A. K. Soe et al. [12] described motion blur as clear streaking of fast movement of an objects in a motionless image.

Neetin kumar et al. [13] find out the true value of PSF applying reverse propagation algorithm for several iteration applied in deblurring method, before applying deconvolution algorithm on blurred image.

D. Singh et al.[14] described the causes of blurring of the digital image, for example motion at the time of capture process, having large exposure duraton, using wide angle lens, etc.

G. Anil [15] described that Gaussian filter blends exact count of pixels incrementally, followed by a bell-shaped 
curvature. The blurring is concentrated in the middle and fluff at the border.

S. Saadi et al. [16] proposed the training of the neural network applying a group optimization algorithm referred as Artificial Bees Colony (ABC).

Swati Sharma et al. [17] proposed a method that first capture DWT of blurred image followed by division into four subfrequencies bands.

Shamik Tiwari et al. [18] described that in the deficiency of noise, the Wiener filter diminishes to the ideal converse filter. Weiner filter reduces the average square error between the proposed random method and the required method.

Dejee Singh et al. [19] simulated motion blur effects in an image applying motion filter in a particular way then the consequented image will seems in motion.

Al-Amri et al. [20] described the Point Spread Function (PSF) is the level at which an optical scheme blurs a point of light.

Ashwini et al. [21] presents an incremental process means it uses several iteration in the deconvblind() method to deblur an image. Result of blind deconvolution is efficient than other present methods.

Ding et al. [22] proposed a method in which the Pixels in the blurred image are represented in terms of the point spread function and the latent (sharp) image.

El-Henawy et al. [23] performed some experiments to assess debluring methods in terms of performance, blur type, Peak Signal to Noise Ratio and structural similarity.

Aarpna et al.[24]divided image pixels into two types: Inliers and Outliers. Inliers pixels are enclosed under restricted dynamic region of camera sensor. Outlier's pixels are nongaussian noise that causes humming artifacts in the consequent retrieved image. This type of noise cannot be handled by existing methods.

Prodip et al. [25] applied Weiner filter using fast Hartley transform (FHT) to increase the pace of deblurring procedure.

Sudha Yadav et al. [26] analyzed different image deblurring methods and their study of performance.

Yu-Wing Tai et al. [27] discussed the issues of modeling and restoring image blur induced by camera movement that follows a projective motion path. They proposed a new Projective Motion Blur Model that consider the blurred image as an combination of a clear scene under a chain of projective transformations that describe the camera's path. The advantages of this motion blur model is that it closely represents spatially changing motion blur devoid of the requirement for clear blurs kernels or having to section the image into local regions with the same spatially invariant blur. A basic constraint to their method is that the high frequency details that have been vanished in the motion blur procedure cannot be restored.

\section{UNDERSTANDING DEBLURRING}

This section gives a general idea on deblurring methods. The segment includes the following points:

\subsection{Reason of Blurring}

The reasons of blurring, of an image are as following:

3.1.1Motion at the time image capture procedure using camera or elongated disclosure duration is applied when image capture.

3.1.2Out-of-focus objects, utilize of a wide-angle lens, environmental disturbance, or a tiny exposure duration that decrease photons captured count.

3.1.3Spreaded optical deformation in confocal microscopy

\subsection{Model of Deblurring}

Description of a blurred image can be given by the following equation

$\mathbf{a}=\mathbf{D b}+\mathbf{u}$, where

$\mathbf{a}$ :The degraded image

D: The degradation operator is often denoted as point spread function (PSF). The PSF denotes the degree at which an optical process spreads a point of light.

b : The original true image

u: induced noise, introduced at the time of image attainment, that blurred the image.

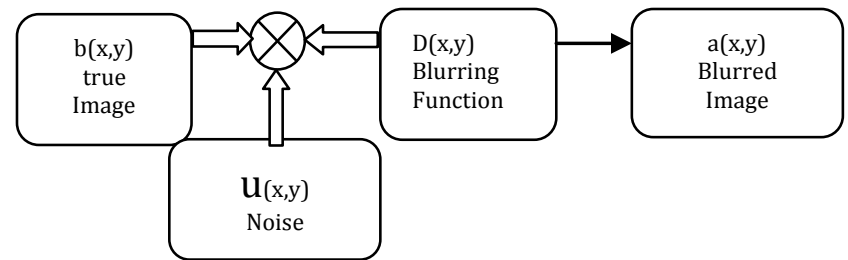

Fig-1: Deblurring Model

\section{DEBLURRING TECHNIQUES}

There are several image restoration methods in image processing. In this section we classified four basic methods as given below:

\subsection{Deblurring with the Wiener Filter}

The converse filtering is a restoration method for deconvolution. It is possible to restore the image by converse filtering if the image is blurred by an identified low pass filter. However, converse filtering is extremely susceptible to additive noise. Algorithm can be developed for every kind of blurring by restoring one kind of blurr at a time and finally combine all of them. The Wiener filter works on an optimal trade-off between converse filtering and noise reduction. It reduces the additive noise as well as deblurring simultaneously.

For mean square error, the Wiener filter is optimal. In other words, in the process of inverse filtering and noise smoothing, it minimizes the overall mean square error. The Wiener filter is a linear assessment of the novel image. The method is relying on a stochastic construction. The orthogonality law implies that the Wiener filter in Fourier domain may be defined as follows:

$$
\mathbf{W}\left(\mathbf{f}_{1}, \mathbf{f}_{2}\right)=\frac{\mathbf{H}^{*}\left(\mathbf{f}_{1}, \mathbf{f}_{12}\right) \mathbf{S}_{\mathbf{x x}}\left(\mathbf{f}_{1}, \mathbf{f}_{2}\right)}{\left|\mathbf{H}\left(\mathbf{f}_{1}, \mathbf{f}_{2}\right)\right|^{2} \mathbf{S}_{\mathrm{xx}}\left(\mathbf{f}_{1}, \mathbf{f}_{2}\right)+\mathbf{S}_{\eta \eta}\left(\mathbf{f}_{1}, \mathbf{f}_{2}\right)}
$$

Where $\operatorname{Sxx}\left(\mathrm{f}_{1}, \mathrm{f}_{2}\right), \operatorname{S\eta \eta }\left(\mathrm{f}_{1}, \mathrm{f}_{2}\right)$ power spectra of the original image and the additive noise respectively, and $H\left(f_{1}, f_{2}\right)$ is the blurring filter. It is very easy to find that the Wiener filter has 
two separate components, a converse filtering component and a noise reduction component. It not only deconvoled by converse filtering but also reduces the noise with a compression process.

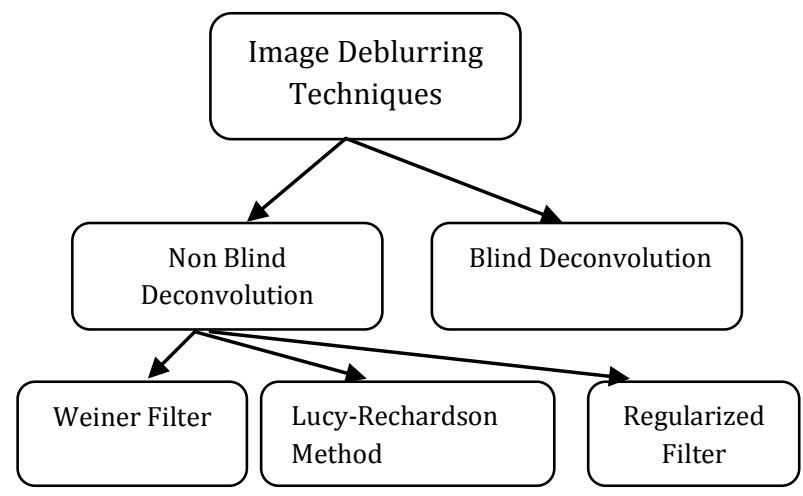

Fig 2: Image Restoration Techniques classification

\subsection{Deblurring with the Lucy-Richardson Algorithm}

According to [27], given the motion blurred image B, the clear image I is computed by Bayesian estimation. The pixel value $\mathrm{I}(\mathbf{x})$ is computed according to pixels values $\mathrm{B}(\mathbf{y})$ in the blurry image by the following formula:

$$
P\left(I_{x}\right)=\sum_{y} P\left(\frac{I_{x}}{B_{Y}}\right) P\left(B_{Y}\right)
$$

Where $P\left(\frac{I_{x}}{B_{Y}}\right)$ can be computed by Bayes's rule.

Use the accelerated, damped, Lucy-Richardson algorithm to deblur an image using the deconvlucy function. When you have very less knowledge about additive noise and you know the PSF in an image then this method can be effective. The deconvlucy method has maximum likelihood to the original Lucy-Richardson algorithm that deal with typical image restoration process. Applying these adaptations, you can:

- Decrease the effect of noise amplification on image restoration.

- Explanation for irregular image quality.

- Deal with camera read-out and background noise.

- Improve the restored image resolution by subsampling

\subsection{Blind Deconvolution Deblurring Algorithm}

Deconvblind method can restore an image by applying the blind deconvolution algorithm. The algorithm optimizes the probability that the resultant image, when convolved with the resultant PSF, is an example of the blurred image, it is assumed the Poisson noise statistics. The blind deconvolution method can be used efficiently if there is not any knowledge about the blurring and noise. The deconvblind method deblurres the image and the PSF at the same time, using a recursive method like the accelerated, damped Lucy-Richardson algorithm.

The deconvblind method, is same as the deconvlucy method, applies many adaptations to the original Lucy-Richardson maximum possibility algorithm that deals with typical image deblurring process. Using these adaptations, you can
- Diminish the outcome of noise on the restoration

- Explanation for irregular image quality.

- Deal with camera read-out noise

\subsection{Regularized filter}

Although the Wiener filtering is the optimal trade-off of inverse filtering and noise smoothing, in the case when the blurring filter is singular, the Wiener filtering actually amplify the noise. This suggests that a denoising step is needed to remove the amplified noise. Wavelet-based denoising scheme, a successful approach introduced recently by Donoho, provides a natural technique for this purpose. Therefore, the image restoration contains two separate steps: Fourier-domain inverse filtering and wavelet-domain image denoising.

Donoho's approach for image restoration improves the performance; however, in the case when the blurring function is not invertible, the algorithm is not applicable Furthermore, since the two steps are separate, there is no control over the overall performance of the restoration. The idea is simple: employ both Fourier-domain Wiener-like and wavelet-domain regularization. The regularized inverse filter is introduced by modifying the Wiener filter with a newintroduced parameter:

$G_{\alpha}=\frac{H^{*} S_{x x}}{|H|^{2} S_{x x}+\alpha S_{\eta \eta}}$

The parameter $\alpha$ can be optimally selected to minimize the overall mean-square error.

\subsection{2-D order-statistic filtering}

Order Statistics filters are nonlinear spatial filters which are based on ordering the pixels contained in an image. Usually, sliding window technique $[4,5]$ is employed to perform pixel-by-pixel operation in a filtering algorithm. The local statistics obtained from the neighbourhood of the centre pixel gives a lot of information about its expected value. If the neighbourhood data are ordered (sorted), then ordered statistical information is obtained. If this order statistics vector is applied to a finite impulse response (FIR) filter, then the overall scheme becomes an order statistics (OS) filter $[1,6]$. They are differentiated based on how they choose the values in the sorted list.

\section{Minimum and Maximum Filter}

The minimum filter selects the smallest value within the pixel values and maximum filter selects the largest value within of pixel values. This is accomplished by a procedure [7] which first finds the minimum and maximum intensity values of all the pixels within a windowed region around the pixel. If the intensity of the central pixel lies within the intensity range spread of its neighbours, it is passed on to the output image unchanged. However, if the central pixel intensity is greater than the maximum value, it is set equal to the maximum value; if the central pixel intensity is less than the minimum value, it is set equal to the minimum value. The minimum and maximum filters are represented as follows:

\section{Median filter}

The median filter considers each pixel in the image in turn and looks at its nearby neighbours to decide whether or not it is representative of its surroundings. Instead of simply replacing the pixel value with the mean of neighbouring pixel values, it replaces it with the median of those values. The median is calculated by first sorting all the pixel values 
from the surrounding neighbourhood into numerical order and then replacing the pixel being considered with the middle pixel value $[6,7]$. (If the neighbourhood under consideration contains an even number of pixels, the average of the two middle pixel values is used.)

\section{Alpha Trimmed Mean Filter}

The alpha-trimmed mean (ATM) filter $[4,7]$ is based on order statistics and varies between a median and mean filter. It is so named because, rather than averaging the entire data set, a few data points are removed (trimmed) and the remainders are averaged. The points which are removed are most extreme values, both low and high, with an equal number of points dropped at each end (symmetric trimming). In practice, the alpha-trimmed mean is computed by sorting the data low to high and summing the central part of the ordered array. The number of data values which are dropped from the average is controlled by trimming parameter alpha which is being expressed as:

\subsection{Median filter}

In doing median filtering, we are computing running medians. From one output picture element to the next, the $m$ $\mathrm{x} \mathrm{n}$ window moves only one column. To get the numbers in the new window from those in the preceding window, we throw away $\mathrm{n}$ points and add in $\mathrm{n}$ new points. The remaining $\mathrm{mn}-2 \mathrm{n}$ numbers are unchanged. To take advantage of this, a fast median filtering algorithm is developed which is based on storing the gray level histogram of the mn picture elements (pels) in the window, and updating it as the window moves. The algorithm consists of the following steps:

Step I: Set up the gray level histogram of the first window and find the median. Also, make the count, ltmdn of the number of pels with gray level less than the median.

Step 2: Move to the next window by deleting the leftmost column of the (previous) window and adding one column to the right. The histogram is updated. So is the count ltmdn. Now ltmdn stores the number of pels in the current window having gray levels less than the median of the previous window.

Step 3: Starting from the median of the previous window, we move up/down the histogram bins one at a time if the count ltmdn is not greaterlgreater than [number of pels in a window divided by 21 and update the count ltmdn until the median bin is reached.

Step 4: Stop if the end of the line is reached. Otherwise go to Step 2.

\section{RESULTS}

To estimate the efficiency of various image deblurring techniques, the quality of the underlying image is measured. There are two kind of method that can be applied to estimate the quality: subjective method and objective method. Subjective method measure the user's contentment or observation without estimating a numeric values through an explicit method. This kind of metrics is the more precise because it is directly accommodate various user needs. The type of metrics is the objective metrics which apply an explicit formula to compute a numeric value. There are two kinds of objective metrics which are commonly used in the assessment of image deblurring methods: Peak Signal-ToNoise Ratio (PSNR).

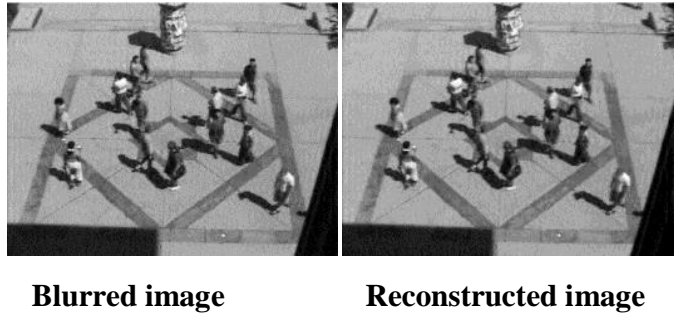

Fig 3: Deblurring using Weiner Filter in combination of Median filter

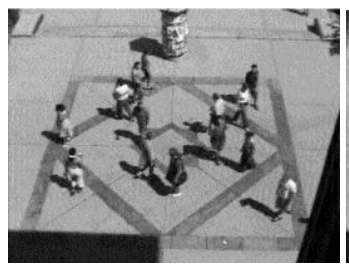

Blurred image

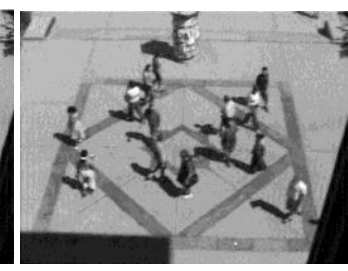

Reconstructed image
Fig 4: Deblurring using Weiner Filter in combination of 2-D order-statistic filtering

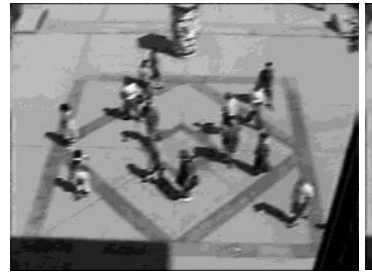

Blurred image

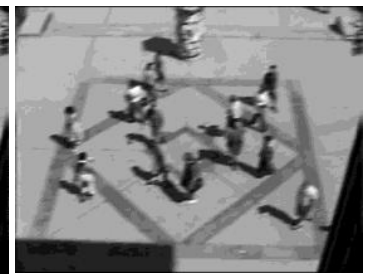

Reconstructed image
Fig 5: Deblurring using Lucy-rechardson Filter in combination of Median filter

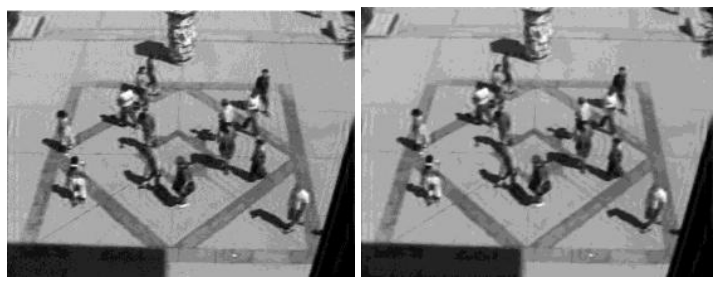

Blurred image

Reconstructed image

Fig 6: Deblurring using Lucy-rechardson Filter in combination of 2-D order-statistic filtering

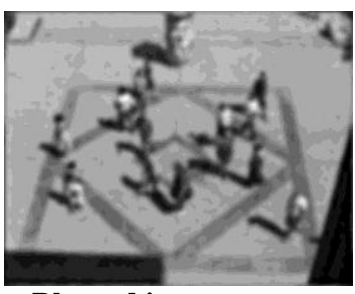

Blurred image

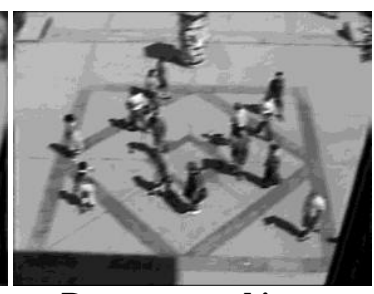

Reconstructed image
Fig 7: Deblurring using Blind deconvolution Filter in combination of 2-D order-statistic filtering 


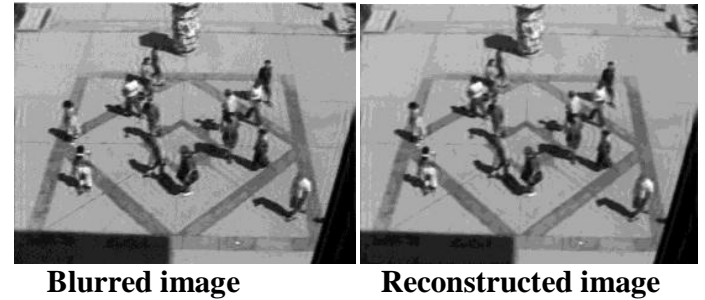

Fig 8: Deblurring using Blind deconvolution Filter in combination of median filter

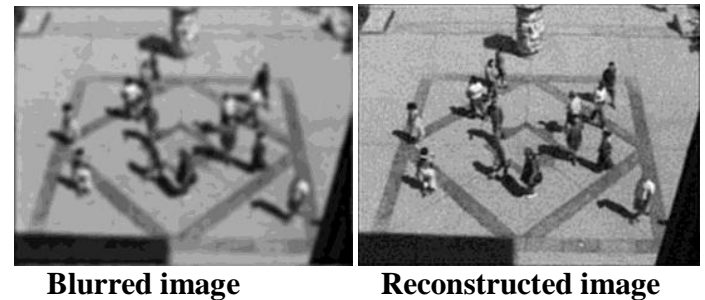

Fig 8: Deblurring using Regularized Filter

PSNR is used to estimate the quality of image in $\mathrm{dB}$ (decibels). PSNR between two images (deblurred image and original image) indicates how distant the two images are equal. Fig-3, fig-4 and fig-5 show the outcomes of subjective measures and table- 1 shows the outcomes of objective measures.

Table 1: of Debluring methods performance analysis

\begin{tabular}{|c|c|c|c|}
\hline $\begin{array}{l}\text { S. } \\
\text { No. }\end{array}$ & Method Name & Blur Type & PSNR \\
\hline 1 & $\begin{array}{l}\text { Weiner filter with } \\
\text { Median Filter }\end{array}$ & Gaussian blur & 29.581 \\
\hline 2 & $\begin{array}{l}\text { Weiner filter with } \\
2 \text {-D order-statistic } \\
\text { filtering }\end{array}$ & Gaussian blur & 29.273 \\
\hline 3 & $\begin{array}{l}\text { Lucy-Richardson } \\
\text { with Median } \\
\text { Filter }\end{array}$ & Gaussian blur & 31.625 \\
\hline 4 & $\begin{array}{l}\text { Lucy-Richardson } \\
\text { with 2-D order- } \\
\text { statistic filtering }\end{array}$ & Gaussian blur & 29.857 \\
\hline 5 & $\begin{array}{l}\text { Blind } \\
\text { deconvolution } \\
\text { with Median } \\
\text { Filter }\end{array}$ & $\begin{array}{l}\text { Gaussian and } \\
\text { Motion }\end{array}$ & 23.196 \\
\hline 6 & $\begin{array}{l}\text { Blind } \\
\text { deconvolution } \\
\text { with 2-D order- } \\
\text { statistic filtering }\end{array}$ & $\begin{array}{l}\text { Gaussian and } \\
\text { Motion }\end{array}$ & 23.261 \\
\hline 7 & Regularized Filter & & 26.514 \\
\hline
\end{tabular}

\section{CONCLUSIONS}

Several methods have been developed by various researchers for image deblurring or image restoration. Till now, image deblurring is a challenging issue. By analyzing various methods, It is our conclusion that the category of Non-blind methods, wiener filter give worst performance, its PSNR (peak signal to noise ratio) value is low as compared to other techniques and Lucy-Rechardson Filter method with median filter is good, its PSNR value is high as compared to other methods. Lucy-Rechardson Filter with median filter method is gives best result in comparison with non-blind techniques and blind deconvolution method. Future scope is to design a hybrid technique to get better result.

\section{REFERENCES}

[1] J. Biemond, R. L. Lagendijk, and R. M. Mersereau.1990 "Iterative methods for image deblurring", Proceedings of the IEEE 78, no. 5, PP. 856-883.

[2] C. M. Jubien, and M. E. Jernigan. 1991 "A neural network for deblurring an image." In Communications, Computers and Signal Processing, IEEE Pacific Rim Conference on, PP. 457-460. IEEE.

[3] J. G. Nagy, K. Palmer, and L.Perrone. 2004 "Iterative methods for image deblurring: a Matlab object-oriented approach." Numerical Algorithms 36, no. 1, PP. 73-93.

[4] P. C. Hansen, 2006 J. G. Nagy, and D. P. O'leary. Deblurring images: matrices, spectra, and filtering. SIAM, Philadelphia.

[5] Z. Hongying, P. Qicong, and W. Yadong. 2006, Variational Image Deblurring Using Modified Hopfield Neural Network, In Communications, Circuits and Systems Proceedings.

[6] J.Jiaya. 2007 Single image motion deblurring using transparency. In Computer Vision and Pattern Recognition, 2007. CVPR'07. IEEE Conference on, pp. 1-8. IEEE

[7] A. Beck, and Marc Teboulle. 2009 A fast iterative shrinkage-thresholding algorithm with application to wavelet-based image deblurring. In Acoustics, Speech and Signal Processing, 2009. ICASSP 2009. IEEE International Conference on, pp. 693-696. IEEE.

[8] Jian-Feng Cai, Hui Ji, Chaoqiang Liu and Zuowei Shen, 2009, Blind motion deblurring from a single image using sparse approximation", IEEE.

[9] Ankit Gupta,Michel Cohen, Brian Curless, 2010 Single image deblurring using motion density functions, chapter computer vision Eccv.

[10] D. S. Rao, K. S. Deepthi, and K.M.S. Deep 2011 Application of Blind Deconvolution Algorithm for Image Restoration. International Journal of Engineering Science and Technology (IJEST).

[11] P. Subashini, M. Krishnaveni, and V. Singh 2011 Image Deblurring Using Back Propagation Neural Network, World of Computer Science and Information Technology Journal (WCSIT), ISSN: 2221-0741, Vol. 1, No. 6, 277-282

[12] A. K. Soe, and X. Zhang 2012 A simple PSF parameters estimation method for the de-blurring of linear motion blurred images using wiener filter in OpenCV. In Systems and Informatics (ICSAI), 2012 International Conference on, pp. 1855-1860. IEEE.

[13] Neetin kumar, Dr. Manish shrivastva 2012 Image deblurring using a neural network approaches, IJEIT, vol 2.

[14] D. Singh,R. K. Sahu 2013 A Survey on Various Image DeblurringTechniques, International Journal of Advanced Research in Computer and Communication Engineering, Vol. 2, Issue 12. 
[15] G. Anil, and R. Kumar, 2013, Design and Analysis of an Algorithm for Image Deblurring using Bilateral Filter, International Journal for Science and Emerging Technologies with Latest Trends, Vol. 5, No. 1, PP. 2834

[16] S. Saadi, A. Guessoum, and M. Bettayeb, 2013, ABC optimized neural network model for image deblurring with its FPGA implementation, Microprocessors and Microsystems 37, no. 1 PP. 52-64.

[17] Swati Sharma, Shipra Sharma and Rajesh Mehra, 2013, Image restoration using modified LR algorithm in the presence of Gaussian and motion blur, AEEE, vol 3.

[18] Shamik Tiwari, V. P. Shukla, A. K. Singh, S. R. Biradar, 2013, Review of motion blur estimation techniques, Journal of Image and Graphics Vol. 1, No. 4.

[19] Dejee Singh, Mr R. K. Sahu ,2013, A survey of various image deblurring techniques", IJARCCE vol. 2, issue 12 .

[20] S. S. Al-Amri, and A. S. Ali., 2014,Restoration and Deblured Motion Blurred Images, International Journal of Computer Science Issues (IJCSI) 11, no. 1.

[21] Ashwini M. Deshpande and Suprava Patnaik 2014, Uniform and Non-uniform single image deblurring based on spares representation and adaptive dictionary learning, IJMA, vol. 6.
[22] J. J. Ding, W.-D. Chang, Y. Chen, S.-W Fu, C.-W Chang, and C.-C.Chang. 2014, Image deblurring using a pyramid-based Richardson-Lucy algorithm, In Digital Signal Processing (DSP), 2014 19th International Conference on, PP. 204-209. IEEE.

[23] I. M. El-Henawy, A. E. Amin, Kareem Ahmed, Hadeer Adel, 2014, A Comparative Study On Image Deblurring Techniques, International Journal of Advances in Computer Science and Technology (IJACST), Vol.3 , No.12.

[24] Aarpna Ashok, deepa, 2015, handling noise and outliers in single image Deblurring using L0 Sparsity, vol 4, issue 7.

[25] Prodip Biswas, Abu Sufian Sarkar, Mohammed Mynuddin, 2015, Deblurring images using weiner filter", IJCA vol.109.

[26] Sudha Yadav, Charu jain, Aarti chugh, 2016, Evaluation of Image Deblurring Techniques, International Journal of Computer Applications (0975 8887) Volume 139 - No.12.

[27] Yu-Wing, Tai Ping, Tan Long Gao, Michael S. Brown,2011, Richardson-Lucy Deblurring for Scenes under Projective Motion Path", IEEE Transactions on Pattern Analysis and Machine Intelligence. 